\title{
Incidence, Maternal Risk Factors, Microbiological Profile And Antibiotic Sensitivity Pattern in Neonatal Sepsis A Hospital Based Study At Agartala, Tripura.
}

\author{
Dr.Sanjib K.Debbarma ${ }^{1}$, Dr.Sujit K.Chakrabarti ${ }^{2}$, \\ ${ }^{I}$ Assistant Professor, ${ }^{2}$ Associate Professor Department Of Paediatrics, Agme \& Gbp Hospital, Agartala, \\ Tripura
}

\begin{abstract}
Background: Neonatal sepsis is a clinical syndrome characterized by signs and symptoms of infection with or without accompanying bacteremia in the first month of life. The reported incidence of neonatal sepsis varied from region to region. Drug resistance has been reported from various parts of the world. Epidemiology of neonatal sepsis has not been methodically studied in the north eastern part of the country. The purpose of this study is to provide a, evidence-based approach to the management of neonates with sepsis.

Objective: This study is taken up to find out the incidence of culture confirmed neonatal sepsis, its microbiological profile and antibiotic sensitivity pattern and influence of maternal risk factors on them. Method: In a retrospective record review pattern all intramural culture confirmed neonatal sepsis cases were included and the maternal risk factors, organisms isolated and the antibiogram was recorded and analyzed. Results: The incidence of culture confirmed sepsis cases was 4.1 per 1000 live birth with preponderance to male sex(58\%) and tribal ethnicity(60\%). Fifty eight percent(58\%) of the cases had some or other form of maternal risk factor. Premature delivery, instrumentation and PROM >24 hours were the most frequently occurring maternal risk factors. In the cases with maternal risk factors Klebsiella pneumonia(26.3\%), Escherichia coli(10.5\%) and Pseudomomas sp.(2.6\%) are more frequently isolated whereas, in cases without maternal risk factors Staphylococcus aureus(18.42\%), Klebsiella pneumonia (15.78\%) and coagulase negative Staphylococcus(7.9\%) were frequent isolates. Most isolates had resistance to first line (Ampicillin+ Gentamicin) and second line (Ceftriaxone + amikacin) antibiotics. Klebsiella and E. Coli were mostly sensitive to Chloramphenicol and quinolones, whereas Staphylococcus aureus and coagulase negative Staphylococcus were susceptible to Vancomycin.

Conclusion: Culture confirmed neonatal sepsis occurred among the intramural neonates at a rate of 4.1 per 1000 live birth with male and tribal preponderance. Maternal risk factors were present upto 58\% of the cases. Klebsiella pneumonia, Staphylococcus aureus, Escherichia coli and coagulase negative Staphylococcus were the leading bacterial isolates. Chloramphenicol, quinolones and Vancomycin were effective against most of the isolates.
\end{abstract}

Keywards: Neonatal sepsis, intramural cases, maternal risk factors ,blood culture, bacteriological isolates/agents, antibiogram, Incidence, evidence-based approach.

Abbreviations:National Neonatal Perinatal Database (NNPD), maternal / perinatal risk factors(MRF), Neonatal intensive care unit( NICU), Muller Hington Agar(MHA), premature rupture of membrane(PROM).

\section{Introduction}

Neonatal sepsis is a clinical syndrome characterized by signs and symptoms of infection with or without accompanying bacteremia in the first month of life. It encompasses various systemic infections of the newborn such as septicemia, meningitis, pneumonia, arthritis, osteomyelitis, and urinary tract infections. Superficial infections like conjunctivitis and oral thrush are not usually included under neonatal sepsis.(1) According to the data from National Neonatal Perinatal Database (NNPD) 2002, the overall incidence of neonatal systemic infection has been reported to be $3 \%$ of intramural live births in tertiary care institutions. Septicemia was the commonest clinical category with an incidence of $2.3 \%$ live births. Klebsiella pneumoniae was the most frequently isolated pathogen (32.5\%), followed by Staphylococcus aureus (13.6\%) among the intramural live births. Amongst extramural babies admitted for neonatal problems, Klebsiella pneumoniae was the commonest organism (27\%), followed by Staphylococcus aureus (15\%) and Pseudomonas (13\%). In contrast to western studies GBS was isolated only in $1 \%$ of the cases(2). Early onset sepsis: Early onset sepsis usually presents within the first 72 hours of life.

The source of infection is generally the maternal genital tract. Clinically, neonates usually present with respiratory distress and pneumonia. Presence of some perinatal risk factors has been associated with an increased risk of early onset sepsis. Late onset sepsis: Late onset sepsis usually presents after 72 hours of age. 
The source of infection is either nosocomial or community-acquired and neonates usually present with septicemia, pneumonia or meningitis. Various factors that predispose to an increased risk of nosocomial sepsis include Neonatal Intensive Care Unit(NICU) admissions, low birth weight, prematurity, invasive procedures, parenteral fluid therapy, ventilation and use of stock solutions. Factors that may increase risk of communityacquired late onset sepsis include poor hygiene, poor cord care, bottle-feeding and prelacteal feeds.(1).

However, maternal/perinatal factors like Premature Rupture Of Membrane(PROM) and meconium stained liquor has been found to be strongly associated with positive blood culture in both early and late onset $\operatorname{sepsis}(8)$.

Emerging multiple drug resistance has become a major concern and has been reported from various parts of the world (10). Knowing the local antibiotic sensitivity pattern is of utmost importance both from therapeutic and preventive point of view. The present study is an attempt to evaluate the incidence, microbiological profile and the local antibiotic sensitivity pattern in septic neonates and find out the influence of maternal risk factors on these. A study like this has been taken up for the first time in the north eastern part of country.

\section{Material And Method}

Study Setting: Neonatal ward and NICU of Agartala Government Medical College (AGMC \& GBP Hospital), Agartala, Tripura.

Study Design: Retrospective observational study.

Study Period: January 2015 to December 2015.

Study Sample:

* Case: All neonates admitted in Neonatal ward and NICU who had positive blood culture and/ or positive sepsis screen during the study period.

* Inclusion Criteria: Neonates admitted to nursery with clinical features neonatal sepsis and/or positive sepsis screen during the study period.

* Exclusion Criteria: Neonates admitted to nursery with life threatening congenital malformation, ambiguous genitalia, severe birth asphyxia, Respiratory distress caused by Hyalin Membrane Disease(HMD), pneumothorax etc., haematological diseases like immune haemolytic disease, red blood cell enzyme defects etc.

Study Size: All neonates with clinical features neonatal sepsis and/or positive sepsis screen and/or blood culture positive neonatal sepsis during the study period.

Study Tool: Laboratory registers and case sheets of all neonates with clinical features neonatal sepsis and/or positive sepsis screen and/or blood culture positive neonatal sepsis during the study period admitted in the hospital.

\section{Methodology}

Agartala Govt Medical College hospital is a postgraduate teaching hospital which has a well-equipped NICU and Neonatal Ward with qualified nurses and specialists. In this retrospective observational study laboratory registers case sheets of all neonates with clinical features neonatal sepsis and/or positive sepsis screen and/or blood culture positive neonatal sepsis during the study period admitted in the hospital, were reviewed. Subsequently data from 127 cases who had clinical features neonatal sepsis and/or positive sepsis screen and/or blood culture positive neonatal sepsis were obtained from their respective case sheet records. Clinical features on which neonatal sepsis were suspected were non-specific features like (a) Hypothermia or fever (b) Lethargy, poor cry, refusal to suck (c) prolonged capillary refill time (d) Hypotonia, absent neonatal reflexes (e) Brady/tachycardia (f) Respiratory distress, apnea and gasping respiration (g) Hypo/hyperglycemia etc. and specific features related to various systems like bulging anterior fontanelle, vacant stare, high-pitched cry, excess irritability, stupor/coma, seizures, neck retraction(CNS), poor perfusion, shock(CVS), feed intolerance, vomiting, diarrhea, abdominal distension, hepatomegaly, direct hyperbilirubinemia (GIT), oliguria/aneuria(Renal), bleeding, petechiae, purpura(Haematological), many pustules, abscess, sclerema, mottling(Dermatologicals) etc.(1). All neonates with suspected sepsis had their blood sample sent for culture and antibiogram in the Department of Microbiology maintaining standard protocol. 1- $2 \mathrm{ml}$ of venous blood was used for culture using brain heart infusion broth. Antibiogram of isolates were done by disc diffusion technique using Muller Hington Agar (MHA). Presence of maternal risk factors like Preterm delivery, febrile illness in the mother within 2 weeks prior to delivery, foul smelling and/ or meconium stained liquor amnii, prolonged rupture of membranes $>24$ hours, more than 3 vaginal examinations during labor, prolonged and difficult delivery with instrumentation, perinatal asphyxia etc were obtained from patient's case record sheet. 


\section{Statical analysis.}

Association of all risk factors with culture positivity was obtained by using Fisher's exact test. A p-value of less than 0.05 is considered as significant.

\section{Results And Observation}

In a one year retrospective study from January 2015 to December 2015, 38 culture-confirmed neonatal sepsis cases were present and the number of live birth at that period was 9185. Thus the Incidence of culture confirmed sepsis cases was 4.1 per 1000 live birth. Forty two percent $(42 \%)$ of the cases were among the Bengali community and $58 \%$ of the cases were among the Tribal community. Maternal risk factors were found in $58 \%$ of the cases whereas in $42 \%$ of the cases no maternal risk factors were found. Among the culture positive sepsis cases mortality was found to be $24 \%$.

Table-1. Age \& sex distribution of the cases $(n=38)$

\begin{tabular}{|l|l|l|l|}
\hline Age & Male & Female & Total \\
\hline $0-3$ days & $12(32 \%)$ & $10(26 \%)$ & $22(58 \%)$ \\
\hline$>3-28$ days & $9(24 \%)$ & $7(18 \%)$ & $16(42 \%)$ \\
\hline Total & $21(56 \%)$ & $17(44 \%)$ & $38(100 \%)$ \\
\begin{tabular}{|l|l|l|c|}
\hline \multicolumn{5}{|l|}{ P Value } \\
\hline CULTURE Positive & MRF Present & MRF Absent & 0.0022 \\
\hline CULTURE Negative & 22 & 16 & \\
\hline
\end{tabular}
\end{tabular}

Table-2. Distribution of Maternal Risk Factors among the cases $(n=22)$

\begin{tabular}{|l|l|l|}
\hline Risk Factors & No. of Cases $(\%)$ & p value \\
\hline Premature delivery(<37 wks) & $5(23 \%)$ & 0.0104 \\
\hline Difficult delivery \& instrumentation & $4(18 \%)$ & 0.2088 \\
\hline H/O PROM >24 hours & $3(14 \%)$ & 0.5517 \\
\hline H/O PET & $2(9 \%)$ & 1.0000. \\
\hline H/O Maternal fever within 2 weeks & $2(9 \%)$ & 1.0000. \\
\hline H/O Ante Partum Haemorrhage & $2(9 \%)$ & 1.0000. \\
\hline Foulsmelling/meconium stained liquor & $2(9 \%)$ & 1.0000. \\
\hline H/O maternal Diabetes Malitus & $1(5 \%)$ & 0.4340 \\
\hline H/o maternal Urinary Tract Infection & $1(5 \%)$ & 0.4340 \\
\hline
\end{tabular}

Table-3. Yield of Positive Blood Culture

\begin{tabular}{|l|l|}
\hline No of neonates tested & $\begin{array}{c}\text { No. of positive blood } \\
\text { culture }(\%)\end{array}$ \\
\hline 127 & $38(29.9 \%)$ \\
\hline
\end{tabular}

Table 3a. Association between MRF and culture positivity

Table-4. Distribution of Bacteriological Agents among the cases $(n=38)$.

\begin{tabular}{|l|l|}
\hline Organism & No.of Cases (\%) \\
\hline Klebsiella & $16(42.1 \%)$ \\
\hline Staph Aureus & $10(26.3 \%)$ \\
\hline E.Coli & $5(13.2 \%)$ \\
\hline Coagulase negative Staphylococcus & $3(7.9 \%)$ \\
\hline Pseudomonas & $1(2.6 \%)$ \\
\hline Citrobacter sp. & $1(2.6 \%)$ \\
\hline Enterobacter sp. & $1(2.6 \%)$ \\
\hline Acinetobacter sp. & $1(2.6 \%)$ \\
\hline
\end{tabular}

Table-5. Distribution of Bacteriological Agents V/S Maternal Risk Factors (MRF)

\begin{tabular}{|l|l|l|}
\hline Organism & No.of Cases with MRF (\%) & $\begin{array}{l}\text { No.of Cases without MRF } \\
(\%)\end{array}$ \\
\hline Klebsiella & $10(26.3 \%)$ & $6(15.78 \%)$ \\
\hline Staph Aureus & $3(7.9 \%)$ & $7(18.42 \%)$ \\
\hline E.Coli negative & $4((10.5 \%)$ & $1(2.6 \%)$ \\
\hline $\begin{array}{l}\text { Coagulase } \\
\text { Staphylococcus }\end{array}$ & $0(0 \%)$ & $3(7.9 \%)$ \\
\hline Pseudomonas & $1(2.6 \%)$ & $0(0 \%)$ \\
\hline Citrobacter sp. & $0(0 \%)$ & $1(2.6 \%)$ \\
\hline Enterobacter sp. & $1(2.6 \%)$ & $0(0 \%)$ \\
\hline Acinetobacter sp. & $0(0 \%)$ & $1(2.6 \%)$ \\
\hline
\end{tabular}


Bar Diagram-1. Bacteriological Isolates V/S Maternal Risk Factors (MRF)

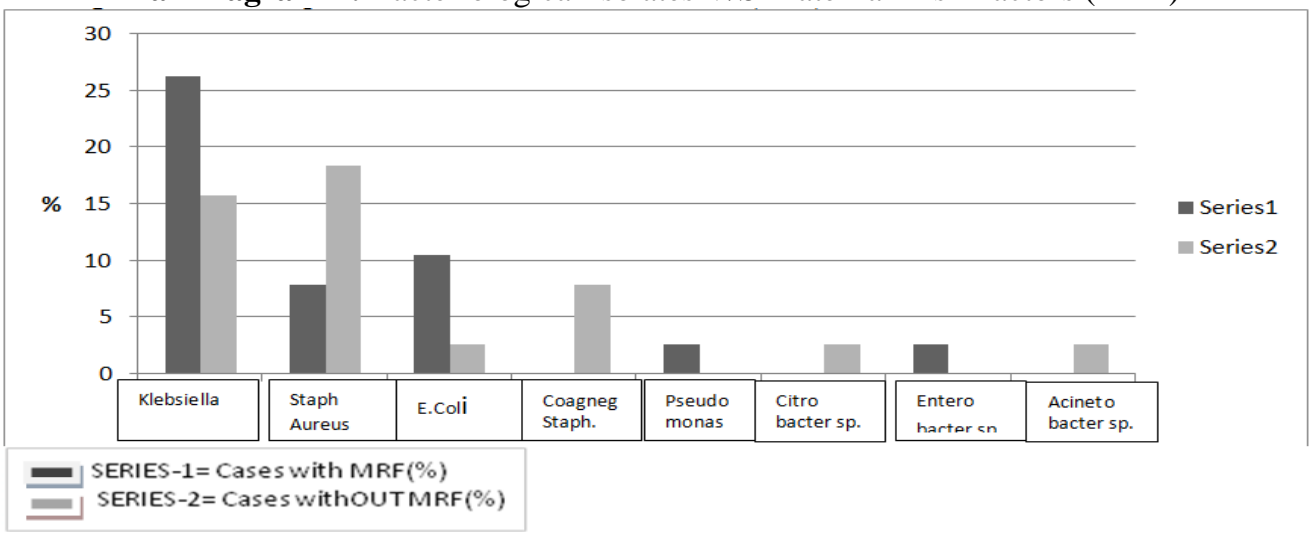

Table-6. Antibiogram of culture isolates:

\begin{tabular}{|c|c|c|c|c|c|c|c|c|c|c|c|c|c|}
\hline $\begin{array}{l}\text { Organi } \\
\text { sm }\end{array}$ & $\begin{array}{l}\text { No. } \\
\text { of } \\
\text { cases }\end{array}$ & $\begin{array}{l}\text { statu } \\
\text { s }\end{array}$ & $\begin{array}{l}\text { Am } \\
\text { pi } \\
\text { cilli } \\
n\end{array}$ & $\begin{array}{l}\text { Ami } \\
k \\
\text { acin }\end{array}$ & $\begin{array}{l}\text { Gent } \\
\mathrm{a} \\
\text { mici } \\
\mathrm{n}\end{array}$ & $\begin{array}{l}\text { Cefo } \\
\text { taxi } \\
m\end{array}$ & $\begin{array}{l}\text { Ceftr } \\
\text { iaxon } \\
\text { e }\end{array}$ & $\begin{array}{l}\text { Ceph } \\
\text { alexi } \\
n\end{array}$ & $\begin{array}{l}\text { Chlora } \\
\mathrm{m} \\
\text { phenico } \\
1\end{array}$ & $\begin{array}{l}\text { Cipro } \\
\text { floxaci } \\
n\end{array}$ & $\begin{array}{l}\text { Vanc } \\
\text { o } \\
\text { mycin }\end{array}$ & $\begin{array}{l}\text { Oflo } \\
\text { xacin }\end{array}$ & $\begin{array}{l}\text { Eryt } \\
\text { hro } \\
\text { myci } \\
\text { n }\end{array}$ \\
\hline \multirow{3}{*}{$\begin{array}{l}\text { Klebsi } \\
\text { ella }\end{array}$} & \multirow[t]{3}{*}{16} & $S$ & & 5 & & & 2 & & 12 & 10 & 2 & 10 & \\
\hline & & $\mathrm{P}$ & 3 & & 3 & 6 & & & 4 & 6 & 10 & 6 & \\
\hline & & $\mathrm{R}$ & 13 & 11 & 13 & 10 & 14 & 16 & & & 4 & & 16 \\
\hline \multirow{3}{*}{$\begin{array}{l}\text { Staph } \\
\text { Aureu } \\
\mathrm{s}\end{array}$} & \multirow[t]{3}{*}{10} & $S$ & 3 & & & & & & 3 & 3 & 10 & 3 & 1 \\
\hline & & $\mathrm{P}$ & & & 2 & & 2 & & 7 & & & & \\
\hline & & $\mathrm{R}$ & 7 & 10 & 8 & 10 & 8 & 10 & & 7 & & 7 & 9 \\
\hline \multirow[t]{3}{*}{ E.Coli } & \multirow[t]{3}{*}{5} & $S$ & & 5 & 5 & & & & 5 & 5 & & 5 & \\
\hline & & $\mathrm{P}$ & 5 & & & 5 & 5 & & & & 5 & & \\
\hline & & $\mathrm{R}$ & & & & & & 5 & & & & & 5 \\
\hline \multirow{3}{*}{$\begin{array}{l}\text { Coagu } \\
\text { lase } \\
\text { negati } \\
\text { ve } \\
\text { Staphy } \\
\text { lococc } \\
\text { us }\end{array}$} & \multirow[t]{3}{*}{3} & $\mathrm{~S}$ & 1 & & & & & & & & 3 & & \\
\hline & & $\mathrm{P}$ & & 3 & & 1 & 1 & & 3 & & & & \\
\hline & & $\mathrm{R}$ & 2 & & 3 & 2 & 2 & 3 & & 3 & & 3 & 3 \\
\hline \multirow{3}{*}{$\begin{array}{l}\text { Pseud } \\
\text { omona } \\
\text { s }\end{array}$} & \multirow[t]{3}{*}{1} & $S$ & & 1 & & & 1 & & & 1 & & 1 & \\
\hline & & $\mathrm{P}$ & & & & & & & & & & & \\
\hline & & $\mathrm{R}$ & 1 & & 1 & 1 & & 1 & 1 & & 1 & & 1 \\
\hline \multirow{3}{*}{$\begin{array}{l}\text { Citrob } \\
\text { acter } \\
\text { sp. }\end{array}$} & \multirow[t]{3}{*}{1} & $S$ & & & & & & & 1 & 1 & & 1 & \\
\hline & & $\mathrm{P}$ & & & & & & & & & & & \\
\hline & & $\mathrm{R}$ & 1 & 1 & 1 & 1 & 1 & 1 & & & 1 & & 1 \\
\hline \multirow{3}{*}{$\begin{array}{l}\text { Entero } \\
\text { bacter } \\
\text { sp. }\end{array}$} & \multirow[t]{3}{*}{1} & $S$ & & & & & & 1 & & 1 & & 1 & \\
\hline & & $\mathrm{P}$ & & 1 & 1 & & & & & & & & \\
\hline & & $\mathrm{R}$ & 1 & & & 1 & 1 & & 1 & & 1 & & 1 \\
\hline \multirow{3}{*}{$\begin{array}{l}\text { Acinet } \\
\text { obacte } \\
\text { r sp. }\end{array}$} & \multirow[t]{3}{*}{1} & $S$ & & 1 & 1 & & & & & 1 & & 1 & \\
\hline & & $\mathrm{P}$ & & & & & & & & & & & \\
\hline & & $\mathrm{R}$ & 1 & & & 1 & 1 & 1 & 1 & & 1 & & 1 \\
\hline
\end{tabular}

Pie diagram-1 : Showing short term outcome

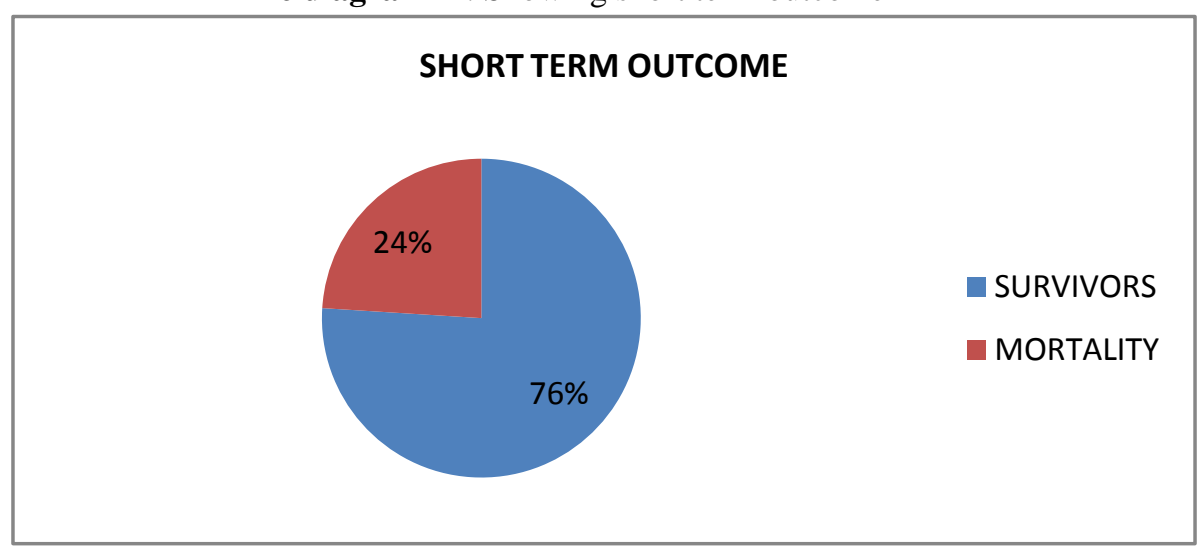




\section{Discussion}

The present study was a retrospective observational study for a period of one year. During the study period 38 culture confirmed neonatal sepsis cases were found after screening 127 neonates and the incidence of culture confirmed sepsis cases was 4.1 per 1000 live birth. As per NNPD report 2002-03 the incidence of systemic infection was $3.0 \%$ (2).But the reported incidence of neonatal sepsis varied from region to region. S Vergnano et al in their article "Neonatal sepsis: an international perspective", reported that incidence of neonatal sepsis varies from 7.1 to 38 per 1000 live births in Asia, from 6.5 to 23 per 1000 live births in Africa, and from 3.5 to 8.9 per 1000 live births in South America and the Caribbean. By comparison, rates reported in the United States and Australasia range from 1.5 to 3.5 per 1000 for EOS sepsis and up to 6 per 1000 live births for LOS sepsis, a total of 6-9 per 1000 for neonatal sepsis.(5). Greenberg et al in a study of 1229 cases of hospital and community-acquired neonatal sepsis found that the rates of neonatal sepsis was $3.2 / 1000$ live births.(9). In our study incidence of culture confirmed sepsis cases was 4.1 per 1000 live birth. This wide variation might have aroused out of difference of regiona variation, study design and genetic characteristics of the ethnic study group. Furthur in depth study in this issue is warranted. In the present study sex distribution was as Male $56 \%$ and female 44. \%.In NNPD report 2002-03 the sex distribution was as follows: males 67.2\%; females $32.7 \%$.(2). This difference is not statistically significant( $\mathrm{SE}=7.5, \mathrm{RD}=1.5, \mathrm{p}>0.05)$. Number of cases were more in the tribal population (58\%) in comparison to the Bengali population (42\%) and for their socio-cultural and socio-economic factors could be responsible for these aberrations.

In our study $58 \%$ of the cases had some or other form of maternal risk factor. Presence of a maternal risk factor and positive blood culture were significantly related( $p=0.0022)$. Schacht A, Zwickau $S$ S et al in their study entitled, "Risk Factors and Opportunities for Prevention of Early-onset Neonatal Sepsis: A Multicenter Case-Control Study" (4) found slightly higher occurrence (79\%) of maternal risk factors in EOS. The difference is significant $(\mathrm{SE}=7.5, \mathrm{RD}=2.4, \mathrm{p}<0.05)$. This difference might have emerged because of multicentricity of the study and restriction of observations to EOS only. Kayange et al. in another study entitled "Predictors of positive blood culture and deaths among neonates with suspected neonatal sepsis in a tertiary hospital, Mwanza- Tanzania"(2010) observed that Perinatal factors like PROM and meconium stained liquor were strongly associated with positive blood culture in both early and late onset sepsis $(p \leq 0.001)$. Gestational age and small gestational age by birthweight were not found to influence rate of positive blood culture in both early and late onset neonatal sepsis. In the present study pre-term delivery was significantly associated with culture positivity $(\mathrm{p}=0.0104)$. In our study out of 127 sepsis suspects 38 had positive blood culture $(29.9 \%)$. Kohli-kochhar R, Omuse J and Revati G in their study entitled "A ten year review of neonatal bloodstream infection in a tertiary private hospital, Kenya" had a culture yield of $23 \%$ ( $p>0.05)$. Our rate of culture positivity is almost similar to their positivity rate .(7)

As per NNPD 2002-03 report Klebsiella pneumoniae was the most frequently isolated pathogen (32.5\%), followed by Staphylococcus aureus (13.6\%) and Escherchia coli (10.6\%).(2) Greenberg d, Shinwel et al in a study of 1229 cases of hospital and community-acquired neonatal sepsis isolated a total of 251 organisms in Southern Israel. Fifty-seven of all isolates were Gram-negative organisms (mainly Klebsiella pneumoniae (20\%) and Escherichia coli (16\%)). Gram-positive organisms were isolated in $41 \%$ of cases. Although E. coli was the most frequently recovered Gram-negative pathogen in community-acquired late onset sepsis, Klebsiella and Enterobacter spp. represented the most commonly isolated Gram-negative organisms in nosocomial late onset sepsis. All Staphylococcus aureus isolates recovered in late onset sepsis were nosocomial. The incidence of Streptococcus agalactiae was 3 times higher in early onset sepsis than in late onset sepsis.(9). In the present study Klebsiella pneumonia(42.1\%), Staphylococcus aureus(26.3\%), Escherichia coli(13.2\%) and coagulase negative Staphylococcus $(7.9 \%)$ were the leading cause. In the cases with maternal risk factors Klebsiella pneumonia, Escherichia coli and Pseudomomas sp. are more frequently isolated whereas in cases without maternal risk factors Staphylococcus aureus, coagulase negative Staphylococcus(7.9\%) and Klebsiella pneumonia were frequent isolates. These present findings are in concordance with most of the similar studies.

Most interestingly antibiotic sensitivity pattern of the of the most frequently occurring isolates had resistance to first line (Ampicillin+ Gentamicin) and second line( Ceftriaxone + amikacin) antibiotics. Klebsiella and E. Coli were sensitive to Chloramphenicol and quinolones, whereas Staphylococcus aureus and coagulase negative Staphylococcus were susceptible to Vancomycin. Emerging multiple drug resistance has also been reported in other parts of the world. The data of Orrett and Shurland(13) from Trinidad show 85\% of S aureus are resistant to ampicillin, and Pseudomonas had $76.6 \%$ resistance to ceftazidime and $72.1 \%$ resistance to gentamicin. The study of Joshi et al(11) from India shows a predominance of Gram negative bacteraemia $(67.2 \%)$ in their series, which had $25-75 \%$ resistance to cephalosporins, $68-78 \%$ resistance to piperacillin, and 23-69\% resistance to gentamicin. The present study reflected similar phenomenon which will have an impact on the present day empirical antibiotic therapy regimen.

Neema Kayange et al in their prospective cross sectional study involving 300 neonates admitted at BMC neonatal unit, Tanzania between March and November 2009 found the mortality rate of neonatal sepsis 
was 19\%. Rajlakshmi Viswanathan and her team in their study entitled Profile of Neonatal Septicaemia at a District-level Sick Newborn Care Unit, West Bengal found one-third of babies, whodeveloped culture-positive sepsis, died. Lydia Mudzikati \& Angela Dramowski (2015) in their study entitled " Neonatal septicemia: prevalence and antimicrobial susceptibility patterns of common pathogens at Princess Marina Hospital, Botswana, Southern Africa found 117 of 223 cases studied were culture positive, giving a positivity rate of $52.61 \%$., Out of 117 neonates $38.94 \%$ were expired. In our study out of 38 culture positive cases 9 cases expired giving a mortality rate of $24 \%$. Such a variation in the mortality rate is likely to be multifactorial. Factors like infrastructural variation, genetic predisposition and variation in sample size might likely to have influenced the mortality rate.

\section{Conclusion}

The Incidence of culture confirmed sepsis cases was 4.1 per 1000 live birth with slight preponderance to male sex. Number of cases was more in the tribal population in comparison to the Bengali population. The yield of positive blood culture was $29.9 \%$. In our study $58 \%$ of the cases had some or other form of maternal risk factor $(\mathrm{P}<0.001)$. History of premature delivery, difficult delivery \& instrumentation and PROM >24 hours were the most frequently occurring maternal risk factors. Klebsiella pneumonia(42.1\%), Staphylococcus aureus(26.3\%), Escherichia coli(13.2\%) and coagulase negative Staphylococcus(7.9\%) were the leading bacterial isolates. In the cases with maternal risk factors Klebsiella pneumonia, Escherichia coli and Pseudomomas sp. are more frequently isolated whereas in cases without maternal risk factors Staphylococcus aureus, coagulase negative Staphylococcus(7.9\%) and Klebsiella pneumonia were frequent isolates. Antibiotic sensitivity pattern of the of the most frequently occurring isolates had resistance to first line (Ampicillin+ Gentamicin) and second line( Ceftriaxone + amikacin) antibiotics. In this study Klebsiella and E. Coli were mostly sensitive to Chloramphenicol and quinolones, whereas Staphylococcus aureus and coagulase negative Staphylococcus were susceptible to Vancomycin.. However, this was a retrospective observational study. Therefore, further prospective studies including control groups on the same is required to be taken up in the interest of the newborn of this region.

\section{References}

[1]. Sankar MJ, Agarwal A, Deorari AK, et al. Sepsis in the newborn. Indian J Pediatr. 2008 Mar;75(3):261-6.

[2]. National Neonatal Perinatal Database. [Internet]. NNPD report 2002-03 [cited 2010 Sep 22]. Available from: http://www.newbornwhoccorg/pdf/nnpd_report_2002-03 PDF; 2005.

[3]. Bhutta ZA, Naqvi SH, Muzaffar T, Farooqui BJ.. Neonatal sepsis in Pakistan. Presentation and pathogens. Acta Paediatr Scand 1991;80:596-601.

[4]. Koutouby A, Habib Ullah J. Neonatal sepsis in Dubai United Arab Emirates. J Trop Pediatr 1995;41:177-80.

[5]. S Vergnano, M Sharland, P Kazembe, C Mwansambo, P T Heath. "Neonatal sepsis: an international perspective. Arch Dis Child Fetal Neonatal Ed. 2005;90:F220-F224.

[6]. Karthikeyan G, Premkumar K. Neonatal sepsis: Staphylococcus aureus as the predominant pathogen. Indian J Pediatr 2001;68:7157.

[7]. Kohli-Kochhar R, Omuse G, Revathi G : A ten-year review of neonatal bloodstream infections in a tertiary private hospital in Kenya. . J Infect Dev Ctries. 2011;5(11):799-803.

[8]. Neema Kayange, Erasmus Kamugisha, Damas L Mwizamholya, Seni Jeremiah and Stephen E Mshana. "Predictors of positive blood culture and deaths among neonates with suspected neonatal sepsis in a tertiary hospital, Mwanza- Tanzania", BMC Pediatrics 2010, 10:39

[9]. Greenberg D, Shinwell ES, Yagupsky P, Greenberg S, Leibovitz E, Mazor M, Dagan R. A prospective study of neonatal sepsis and meningitis in southern Israel. Pediatr Infect Dis J 1997;16:768-73.

[10]. Joshi SJ, Ghole VS, Niphadkar KB. Neonatal gram negative bacteremia. Indian J Pediatr 2000;67:27-32.

[11]. Samanta S et al., Risk factors for late onset gram-negative infections: a case-control study, Arch Dis Child Fetal Neonatal Ed . 2011;96:F15-F18.

[12]. Orrett, Fitzroy A.1, Shurland, Simone M.. Neonatal sepsis and mortality in a regional hospital in Trinidad: aetiology and risk factors. Annals of Tropical Paediatrics: International Child Health. 2001; 21(1):20-25.

[13]. Rajlakshmi Viswanathan, Arun K. Singh, Chiranjib Ghosh, Sudipta Dasgupta, Suchandra Mukherjee, and Sulagna Basu. Profile of Neonatal Septicaemia at a District-level Sick Newborn Care Unit. J HEALTH POPUL NUTR 2012; 30(1):41-48.

[14]. Lydia Mudzikati \& Angela Dramowski. Neonatal septicaemia: prevalence and antimicrobial susceptibility patterns of common pathogens at Princess Marina Hospital, Botswana. Southern African Journal of Infectious Diseases, 2015; 30:3, 108-113.

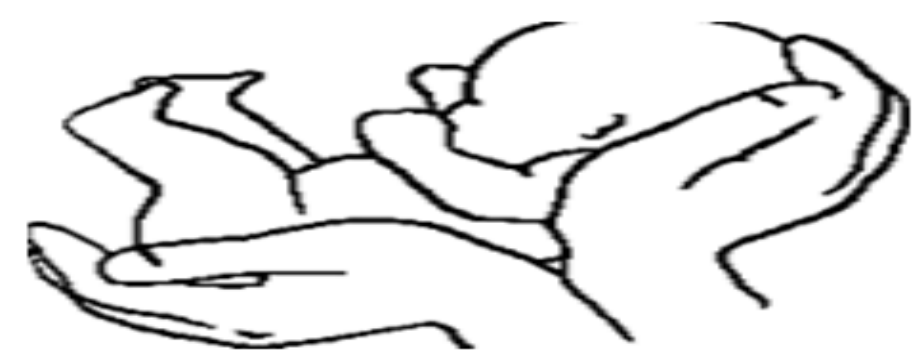

